\title{
CAPACIDADE DE DISPERSÃO DE Trichogramma pretiosum RILEY, 1879 PARA CONTROLE DE Helicoverpa zea (BODDIE, 1850) EM MILHO ${ }^{1}$
}

\author{
L.A. NOGUEIRA DE SÅ \\ Centro Nacional de Pesquisas de Defensivos Agricolas/EMBRAPA, C.P. 69, CEP:13820-000 - Jaguariúna,SP. \\ J.R.P. PARRA; S. SILVEIRA NETO \\ Departamento de Entomologia da ESALQ/USP, C.P. 9 - CEP:13418-900-Piracicaba,SP.
}

RESUMO: Estudou-se, em duas localidades do Estado de Sāo Paulo, a capacidade de dispersão de Trichogramma pretiosum Riley, 1879 na cultura do milho, em relação a ovos de Helicoverpa zea (Boddie, 1850). A dispersão de $T$. pretiosum, 36 horas apos a liberação, foi de 80 a $102 \mathrm{~m}^{2}$. Ocorreu uma relação inversa entre parasitismo e predação natural por insetos, nas duas localidades estudadas. Em função dos resultados obtidos, determinou-se que a liberação dos parasitoides, para controle de $\boldsymbol{H}$. zea, deve ser feita em 100 pontos/ha.

Descritores: Trichogramma pretiosum, capacidade de dispersão, milho, Helicoverpa zea, controle biologico, Zea mays.

\section{DISPERSION CAPACITY OF Trichogramma pretiosum RILEY, 1879 FOR CONTROLLING Helicoverpa zea (BODDIE, 1850) IN CORN}

ABSTRACT: The dispersion capacity of Trichogramma pretiosum Riley, 1879 in relation to Helicoverpa zea (Boddie, 1850) eggs was studied in corn, in two regions, in the State of Săo Paulo. The dispersion capacity of T. pretiosum 36 hours after release ranged from 80 to $102 \mathrm{~m}^{2}$ and an inverse relationship between parasitism and predation occurred for $\boldsymbol{H}$. zea eggs for both localities studied. The results obtained indicate that releases for controlling $H$. zea should be made at 100 points/ha.

Key Words: Trichogramma pretiosum, dispersion capacity, corn, Helicoverpa zea, biological control, Zea mays.

\section{INTRODUÇÃO}

O conhecimento da capacidade de dispersão de um parasitóide é fundamental para se determinar o número de pontos de liberação, por unidade de área, pois deste número depende a maior ou menor eficiência de controle. Assim, KANOUR JUNIOR \& BURBUTIS (1984) verificaram que o parasitismo de Trichogramma nubilale na primeira e na segunda gerações de ovos de Ostrinia nubilalis, variou de 41 a $27 \%$, e que estas taxas de parasitismo decresceram com o aumento da distância do ponto de liberação do parasitóide. Também para Trichogramma maidis, BIGLER et al. (1988) observaram este mesmo comportamento.

O número de pontos de liberação de Trichogramma em milho comercial é variável de 30 pontos/ha na China (HUFFAKER, 1977) a 80 pontos na Alemanha (NEUFFER, 1982).
O objetivo do presente trabalho foi determinar a capacidade de dispersão de Trichogramma pretiosum Riley, $1879 \mathrm{em}$ relação a ovos de Helicoverpa zea (Boddie, 1850) em cultura de milho, conduzida em duas localidades do Estado de São Paulo.

\section{MATERIAL E MÉTODOS}

Para determinar o raio efetivo de ação de Trichogramma pretiosum Riley, 1879 (linhagem 8 da coleção de Trichogramma do Departamento de Entomologia da ESALQ/USP) foram demarcados três círculos concêntricos com raios de 5, 10 e 15 m, em campos de milho, cultivares C505 e AG405, em Santo Antônio de Posse - SP e Piracicaba $S P$, respectivamente. $O$ primeiro círculo teve raio de $5 \mathrm{~m}$ em relação ao ponto central de liberação de $T$. pretiosum e constou de 8 pontos de infestação com ovos de $H$. zea. O círculo de $10 \mathrm{~m}$

\footnotetext{
${ }^{1}$ Parte do trabalho de tese do primeiro autor apresentado à ESALQ/USP, para obtenção do título do Doutor em Ciências.
} 
de diâmetro equivaleu a três vezes a área do círculo de raio $5 \mathrm{~m}$, correspondendo a 24 pontos de infestação. $O$ círculo de $15 \mathrm{~m}$ equivaleu a 5 vezes a área do primeiro círculo, comportando 40 pontos de infestação (Figura 1). Foi demarcada uma área testemunha $\left(900 \mathrm{~m}^{2}\right)$ a $50 \mathrm{~m}$ de distância da parte externa do maior círculo.

Nos dois locais de estudo, foram feitas infestações artificiais de ovos de $H$. zea, obtidos da criação estoque do Laboratório de Biologia do Departamento de Entomologia da ESALQ, em dieta artificial à base de feijão, levedura e germe-detrigo (BURTON, 1969). Utilizaram-se pequenos retângulos de tecido do tipo "fíló" contendo, cada um deles, cerca de 60 ovos.

Estes ovos foram distribuídos em pontos pré-fixados, em número proporcional à área ocupada (Figura 1). Em Piracicaba, os retângulos de filó foram fixados na região do estilo-estigma através de uma haste de arame revestida com cola do tipo "sticky" (Figura 2). Em Santo Antônio de Posse, o "filó" contendo os ovos foi preso às folhas com alfinete sem uso da haste de arame.

O parasitóide foi liberado (cerca de 4.200 adultos) 30 minutos após a infestação artificial, sendo o parasitismo permitido por 36 horas. Após este período, as telas de filó, contendo os ovos, foram coletadas e conduzidas ao laboratório para determinação das porcentagens de parasitismo e predação. Em Santo Antônio de Posse, foram realizadas três infestações artificiais e três liberações de $T$. pretiosum a intervalos de 15 dias. Em Piracicaba foi feita apenas uma liberação de $T$. pretiosum.

A porcentagem de predação foi calculada através da seguinte fórmula:

$$
\operatorname{Predaçao~}(\%)=\frac{I-F}{I} \times 100 \%
$$

Onde:

I - Total de ovos da infestação artificial;

F - Total de ovos recolhidos do campo após a liberação.

As médias das porcentagens de parasitismo e predação foram comparadas pelo teste " $t$ " ao nível de $1 \%$ de probabilidade.

Foram calculadas a distância média de dispersão (DM) e a distância $\left(\mathrm{m}^{2}\right)$ de dispersão $\left(\mathrm{s}^{2}\right)$ pelo modelo de POOLE (1974) para os dois experimentos. Foram calculados o modelo matemático e os coeficientes de correlação $(r)$ e determinação $\left(r^{2}\right)$.

\section{RESULTADOS E DISCUSSÃO}

Observou-se que o parasitismo foi bastante elevado em Piracicaba, considerando-seo período de tempo que os ovos de Helicoverpa zea (Boddie, 1850) permaneceram no campo (36 h). Entretanto, a eficiência do parasitismo decresceu a partir de $5 \mathrm{~m}$ do ponto de liberação (TABELA 1 ). Em Santo Antônio de Posse houve a mesma tendência, ou seja, diminuição de parasitismo a partir de $5 \mathrm{~m}$ do ponto de liberação. Houve correlação inversa, para ambos os locais, entre porcentagem de parasitismo e predação, a qual foi alta e devida, principalmente, a coccinelídeos, crisopídeos e forficulídeos (TABELA 1). Baseando-se no modelo de POOLE (1974), a distância média de dispersão para Santo Antônio de Posse e Piracicaba, variou de 6,31 e $9,36 \mathrm{~m}$, respectivamente. Assim, a área de dispersão do parasitóide correspondeu, para as duas localidades, a 80 e $102 \mathrm{~m}^{2}$, respectivamente (TABELA 2).

Houve uma correlação negativa para ambos os locais, entre distância do ponto de liberação e parasitismo, ou seja, ocorreu diminuição no número de ovos parasitados, à medida que se aumentou a distância do ponto de liberação (TABELA 2 e Figura 3). Resultados semelhantes foram obtidos por BIGLER et al. (1988) para Ostrinia nubilalis e Trichogramma maidis, e por KANOUR JUNIOR \& BURBUTIS (1984) para Trichogramma nubilale, na cultura do milho.

O raio de dispersão de $T$. pretiosum (linhagem 8) observado neste trabalho $(6,31$ a 9,36 m) mostrou-se próximo ao obtido em milho doce por NEUFFER (1982) (10 a $14 \mathrm{~m})$ e por VAN SCHELT \& RAVENSBERG (1991) (10 m) em milho comercial. VAN DEN BERG et al. (1987) verificaram, em pomares cítricos, um parasitismo concentrado em um raio de $15 \mathrm{~m}$ para Trichogramma cryptophlebiae. Em cana-de-açúcar, LOPES et al. (1989) verificaram um raio efetivo de ação de $10 \mathrm{~m}$ para Trichogramma galloi. Estas variações de valores são normais, desde que os fatores que governam a dispersão de parasitóides são complexos, envolvendo aspectos bióticos e abióticos (GOODENOUGH \& WITZ, 1985), incluin-do temperatura, umidade, orvalho, chuva, luz, vento, tamanho do hospedeiro, idade e raça do parasitóide. Como os resultados da pesquisa mostraram um raio efetivo de ação ao redor de 10 $\mathrm{m}$, sugere-se que as liberações de $T$. pretiosum $\mathrm{em}$ milho, devam ser feitas em 100 pontos/ha, para uma distribuição homogênea em toda área. 
TABELA 1 - Porcentagem de predação e do parasitismo de $T$. pretiosum (linhagem 8), em ovos de $H$. zea, colocados a diferentes distâncias de um ponto central de liberação dos parasitóides em milho, em Piracicaba e Santo Antônio de Posse.

\begin{tabular}{ccccc}
\hline \hline Distância & \multicolumn{2}{c}{ Predação (\%) } & \multicolumn{2}{c}{ Parasitismo (\%) } \\
\hline$(\mathrm{m})$ & Piracicaba & Sto. A. da Posse & Piracicaba & Santo A. da Posse \\
\hline 5,0 & $5,83 \pm 4,71$ & $84,03 \pm 5,04$ & $60,21 \pm 3,93 \mathrm{a}^{*}$ & $14,02 \pm 1,21 \mathrm{a}$ \\
10,0 & $4,79 \pm 3,67$ & $86,80 \pm 6,23$ & $39,72 \pm 11,93 \mathrm{~b}$ & $11,36 \pm 0,81 \mathrm{~b}$ \\
15,0 & $6,29 \pm 3,76$ & $90,55 \pm 2,99$ & $13,16 \pm 15,19 \mathrm{c}$ & $5,32 \pm 2,21 \mathrm{c}$ \\
\hline \hline
\end{tabular}

" Médias seguidas da mesma letra, na coluna, não diferem estatisticamente entre si, pelo teste "t", ao nível de $1 \%$ de probabilidade.

TABELA 2 - Distância média (DM) e área de dispersão $\left(\mathrm{s}^{2}\right)$, com os respectivos modelos matemáticos e coeficientes para $T$. pretiosum (linhagem 8 ) em ovos de $H$. zea nas localidades de Piracicaba e Santo Antônio de Posse.

\begin{tabular}{ccc}
\hline \hline Parâmetros & Piracicaba & Santo Antonio da Posse \\
\hline DM $(\mathrm{m})$ & 9,36 & 6,31 \\
$\mathrm{~S}^{2}\left(\mathrm{~m}^{2}\right)$ & 101,74 & 79,76 \\
Modelo Matemático & $y=6,01252-0,0136151 x^{2}$ & $y^{2}=36,0148-8,41531 \quad x$ \\
$\mathrm{R}^{2}(\%)$ & 93,70 & 96,83 \\
$\mathrm{r}$ & $-0,968$ & $-0,984$ \\
\hline \hline
\end{tabular}

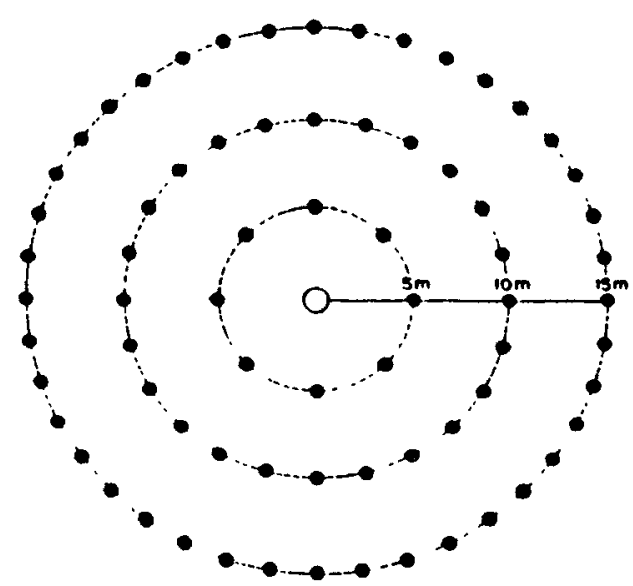

Ponte de liberacton de $r$ arefrosum

Pantos do infestoces ortificlat com posturas de H. 200 .

Figura 1 - Esquema da distribuição de posturas de $H$. zea ao redor de um ponto central de liberação de $T$. pretiosum, para estudo de dispersão.

Sci. agric., Piracicaba, 50(2):226-231, jun./set., 1993 


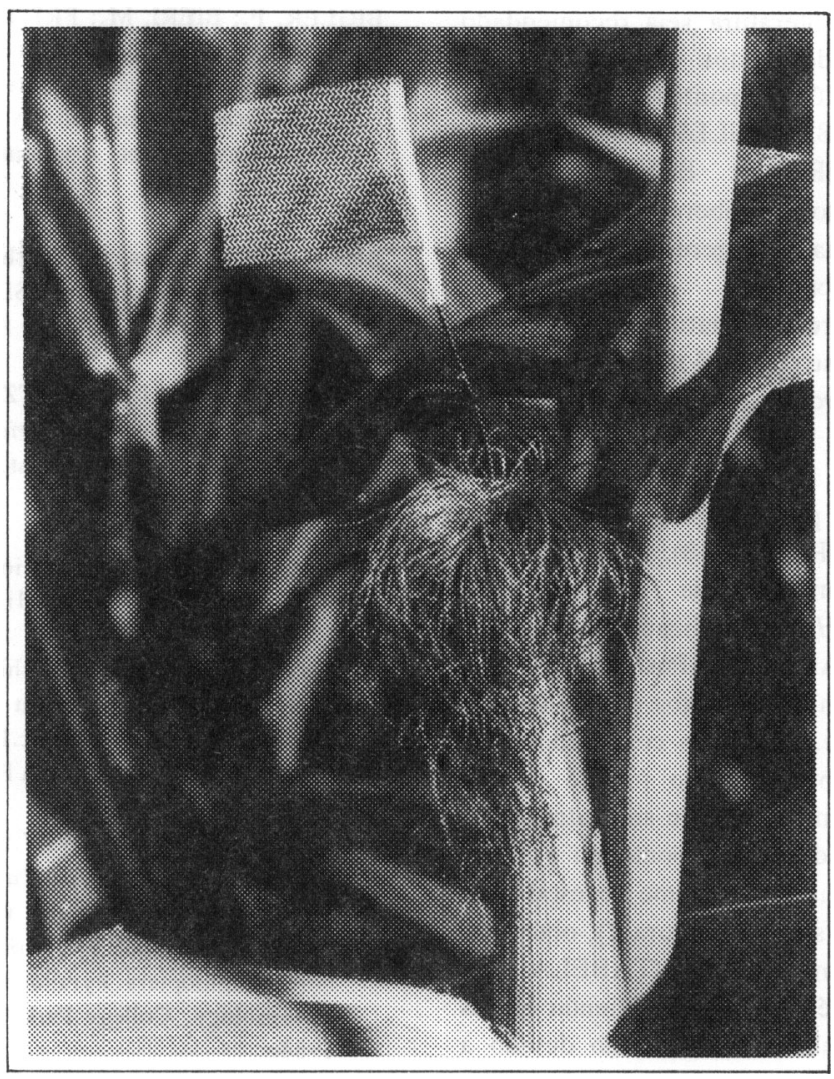

Figura 2 - Retângulos de tecido do tipo "filó" com suporte de arame, contendo ovos de $H$. zea, colocados próximos ao estilo-estigma, para infestação artificial em campo, em Piracicaba.
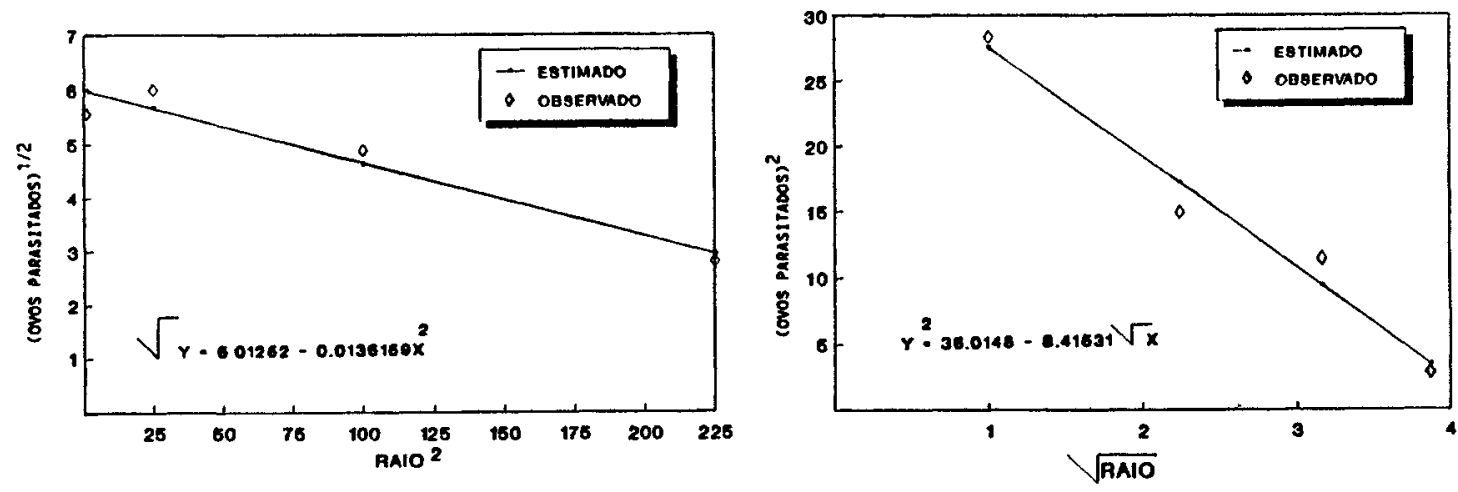

Figura 3 - Relação matemática entre o raio de dispersão de $T$. pretiosum (linhagem 8) e o número de ovos de $H$. zea parasitados, na cultura do milho, em duas localidades. A - Piracicaba; B - Santo Antônio de Posse.

Sci. agric., Piracicaba, 50(2):226-231, jun./set., 1993 
Embora na literatura seja recomendado um número menor de pontos de liberação em milho, ou seja, entre 30 e 80 pontos (HUFFAKER, 1977; NEUFFER, 1982; BIGLER \& BRUNETTI, 1986), RAVENSBERG \& BERGER (1988) propuseram 100 pontos de liberação, para milho doce. Desta forma, sugerem-se pesquisas comparativas neste sentido.

A elevada predação natural verificada em Santo Antônio de Posse $(87,13 \%)$ (Tabela 1) sugere que a avaliação da porcentagem de parasitismo seja feita no máximo 36 horas após a liberação do parasitóide.

Esta alta porcentagem de predação embora comum (LOPES, 1988), pode ter sido influenciada pela metodologia da infestação artificial de ovos de $\boldsymbol{H}$. zea, utilizada na presente pesquisa para Santo Antônio de Posse.

Assim, em Piracicaba utilizando-se metodologia diferente, não mais colocando-se os ovos diretamente sobre o milho (o filo com a postura era fixado com alfinete) e sim, através de pequenos retângulos de "filó", contendo os ovos suportados por uma haste de arame revestida por uma fina camada de "sticky" (Figura 2), obteve-se uma predação bem menor (TABELA 1).

Entretanto,$e$ conveniente salientar que as variações podem também ter ocorrido devido à diferença de localidades e variedades de milho utilizadas. Esta variação pôde ser verificada pela própria porcentagem de parasitismo da testemunha que oscilou de 1 a $9 \%$ em ambas localidades.

\section{CONCLUSÕES}

1. A capacidade de dispersão de $T$. pretiosum com relação a ovos de $H$. zea, em 36 horas, corresponde a uma área de 80 a $102 \mathrm{~m}^{2}$;

2. A liberação de $T$. pretiosum para controle de $H$. zea, deve ser realizada em 100 pontos/ha;

3. Para as duas localidades estudadas há uma relação inversa entre parasitismo e predação.

\section{REFERENCIAS BIBLIOGRÁFICAS}

BIGLER, F.; BRUNETTI, R. Biological control of Ostrinia nubilalis Hbn. by Trichogramma maidis Pint. et Voeg. on corn for seed production in southern Switzerland. Journal of Applied Entomology, Hamburg, v.102, p.303-338, 1986.
BIGLER, F.; BIERI, M.; FRITSCHY, A.; SEIDEL, K. Variation in locomotion between strains of Trichogramma maidis and its impact on parasitism of eggs of Ostrinia nubilalis in the field. Entomologia Experimentalis et Applicata, The Hague, v.49, p.283-290, 1988 .

BURTON, R.L. Mass rearing the com earworm in the laboratory. Washington, USDA Agriculture Research Service, 1969. 8p. (USDA. ARS, 33-134).

GOODENOUGH, J.L.; WITZ, J.A. Modeling augmentative releases of Trichogramma pretiosum. Southwestern Entomologist, College Station, (supl. 8): p.169-189, 1985.

HUFFAKER, C.B. Augmentation of natural enemies in the People's Republic of 'China. In: INTERNATIONAL CONGRESS OF ENTOMOLOGY, 15., Washington, 1976. Biological control by augmentation of natural enemies; proceedings, edited by R.L. Ridgway and S.B. Vinson. New York: Plenum Press, 1977. p.329-340.

KANOUR JUNIOR, W.W.; BURBUTIS, P.P. Trichogramma nubilale (Hymenoptera: Trichogrammatidae) field releases in corn and hypothetical model for control of European corn borer (Lepidoptera: Pyralidae) . Journal of Economic Entomology, College Park, v.77, n.1, p.103-107, 1984.

LOPES, J.R.S. Estudos bioetológicos de Trichogramma galloi (Zucchi, 1988) (Hym., Trichogrammatidae) para controle de Diatraea saccharalis (Fabr., 1794) (Lep., Pyralidae). Piracicaba, 1988. 141p. Dissertação (Mestrado) - Escola Superior de Agricultura "Luiz de Queiróz", Universidade de São Paulo.

LOPES, J.R.S.; PARRA, J.R.P.; JUSTI JUNIOR, J.; OLIVEIRA, N.H. Metodologia para infestaçāo artificial de ovos de Diatraea saccharalis (Fabr., 1794) em cana-de-açúcar visando estudos com Trichogramma spp. Anais da Escola Superior de Agricultura "Luiz de Queir6z", Piracicaba, v.46, n.2, p.375-390, 1989.

NEUFFER, G. The use of Trichogramma evanescens Westw. in sweetcorn fields. A contribution to the biological control of the European corn borer Ostrinia nubilalis Hbn. in south west Germany. In SYMPOSIUM INTERNATIONAL SUR LES TRICHOGRAMMES, 1., 1982, Antibes. Paris: INRA, 1982. p.231-237. (Les Colloques de I'INRA, 9). 
POOLE, R.W. An introduction to quantitative ecology. New York: McGraw-Hill, 1974. 532p. RAVENSBERG, W.J. \& BERGER, H.K. Biological control of the European corn borer (Ostrinia nubilalis Hbn, Pyralidae) with Trichogramma maidis Pintureau and Voegelé in Australia in 1980-1985. In: INTERNATIONAL SYMPOSIUM ON TRICHOGRAMMA AND OTHER EGG PARASITES, 2., 1986, Guangzhou. Paris: INRA, 1988. p.557-564. (Les Colloques de l'INRA, 43).

VAN DEN BERG, M.A.; NEWTON, P.J.; DEACON,

- V.E.; CRAUSE, C. Dispersal of Trichogrammatoidea cryptophlebiae (Hymenoptera: Trichogrammatidae), an egg parasitoid of the false codling moth, Cryptophlebia leucotreta (Lepidoptera: Tortricidae), in an empty habitat. Phytophylactica, Pretoria, v.19, p.515-516, 1987.
VAN SCHELT, J.; RAVENSBERG, W.J. Some aspects on the storage and application of Trichogramma maidis in corn. In: INTERNATIONAL SYMPOSIUM ON TRICHOGRAMMA AND OTHER EGG PARASITOIDS, 3., 1990, San Antonio. Paris: INRA, 1991. p.239-242. (Les Colloques de l'INRA, 56).

Trabalho enviado para publicação em 03.12 .92 Trabalho aceito para publicaçāo em 02.03 .93 